\title{
Myopericarditis in a Young Turkish Woman with Heterozygous Familial Mediterranean Fever
}

\section{Abstract}

Familial Mediterranean Fever (FMF) has many different clinical presentations, the most common of which is fever with serositis. Myopericarditis may occur in patients with FMF. We present the case of a young Turkish female who had an extensive workup for fever, abdominal pain, and mesenteric lymphadenopathy and who later developed myopericarditis. Subsequent genetic testing revealed that the patient was heterozygous for the E148Q mutation in the $M E F V$ gene. The patient was treated with an NSAID initially and was then transitioned to colchicine, with a significant improvement in the frequency of abdominal pain attacks and episodes of high grade fevers and without recurrence of myopericarditis.

Keywords: Familial Mediterranean Fever (FMF); Myopericarditis; Mesenteric lymphadenopathy

\section{Introduction}

Myopericarditis is usually a self-limiting disease, most commonly caused by a viral infection. The most frequent viral etiologies are coxsackie B virus, adenovirus, herpes viruses, hepatitis $C$ virus, parvoviruses and echovirus, among others [1,2]. An underlying autoinflammatory disorder, though uncommon, may also cause myopericarditis. We present a patient with fever, mesenteric lymphadenopathy and abdominal pain who later developed myopericarditis and was subsequently found to be heterozygous for the E148Q mutation in the MEFV gene

\section{Case Presentation}

A 16-year-old girl was referred to the Infectious Diseases clinic at our facility in Dallas, Texas on 1/9/2015 for abdominal pain and fever. The patient had no pertinent past medical history, nor was she taking any medications prior to the onset of her illness. Her symptoms began 6 months earlier when she was working in Missouri as a counselor at a camp for disabled children. At that time, she developed fatigue, fever, sinus pain and nasal drainage. She was treated with amoxicillin/clavulanate for a clinical diagnosis of sinusitis. Most of her symptoms resolved but she still had fatigue. In the interim, she developed intermittent bilateral hip, knee, and ankle pain in addition to intermittent right lower quadrant abdominal pain. She saw a physician in November 2014 and had routine laboratory studies which were unrevealing. She was prescribed another course of amoxicillin/clavulanate, but she continued to have fatigue and later developed high grade fevers without a particular pattern. CT scan of the abdomen revealed mesenteric lymphadenopathy and possible terminal ileitis. The erythrocyte sedimentation rate and C-reactive protein level were reportedly within normal limits. She underwent an upper and lower endoscopy which were normal. An elective appendectomy revealed only chronic inflammation, without acute appendicitis or granulomas.

Case Report
Volume 11 Issue 1 - 2018
Andrew Assaf ${ }^{1 *}$, Jorge Cheirif ${ }^{2}$, Allison
Liddell ${ }^{3}$, Bruce Wall ${ }^{4}$, Rodney Bowman ${ }^{5}$ and
Mark Feldman ${ }^{1}$
${ }^{1}$ Texas Health Presbyterian Hospital of Dallas, USA
${ }^{2}$ Presbyterian Heart and Vascular Group, Texas Health
Physicians Group, USA
${ }^{3}$ Infectious Care, USA
${ }^{4}$ Dallas Nephrology Associates, USA
${ }^{5}$ Department of Radiology, Texas Health Presbyterian Hospital
of Dallas, USA
*Corresponding author: Andrew Davis Assaf, PGY-2
Internal Medicine Resident Physician, Texas Health
Presbyterian Dallas, 8130 Park Ln Apt 1012, USA, 281 743
4849; Email: andrewdavis.assaf@gmail.com
Received: December 30, 2017 | Published: January 16,
2018

The patient developed non-radiating, mid-sternal chest pain that worsened on inspiration with associated shortness of breath on $1 / 20 / 2015$ and presented to our emergency department. On arrival, her blood pressure was 112/56 $\mathrm{mmHg}$, heart rate was regular at 118 beats per minute, temperature was $97^{\circ} \mathrm{F}$ and respiratory rate was 16 breaths per minute. Her pulse oximetry revealed an oxygen saturation of $100 \%$ on room air. Body mass index was $19 \mathrm{~kg} / \mathrm{m}^{2}$. She was a well-developed and well-nourished young female in no acute distress. Her oropharynx was normal. Her cardiac examination revealed sinus tachycardia, normal S1 and S2 with no pericardial knock, friction rub, murmurs, or gallops. A joint examination was normal. There was no rash. Pulmonary and abdominal examinations were also unremarkable.

ECG revealed sinus tachycardia and right axis deviation without ST-T changes (Figure 1). Laboratory studies, summarized in Table 1, revealed a mild normocytic, normochromic anemia and a considerably elevated serum troponin I level. Chest radiography (Figure 2) revealed a non-specific mild left perihilar/lingular area of atelectasis or infiltrate. Given her history of fever, the patient underwent technetium labeled white blood cell (WBC) scanning, prior to the onset of her chest pain, which revealed intense labeled WBC uptake at the left ventricular myocardium consistent with myopericarditis (Figure 3). A transthoracic echocardiogram (Figure 4) revealed normal right and left ventricular (left ventricular ejection fraction of 55-60 \%) and a small pericardial effusion. 


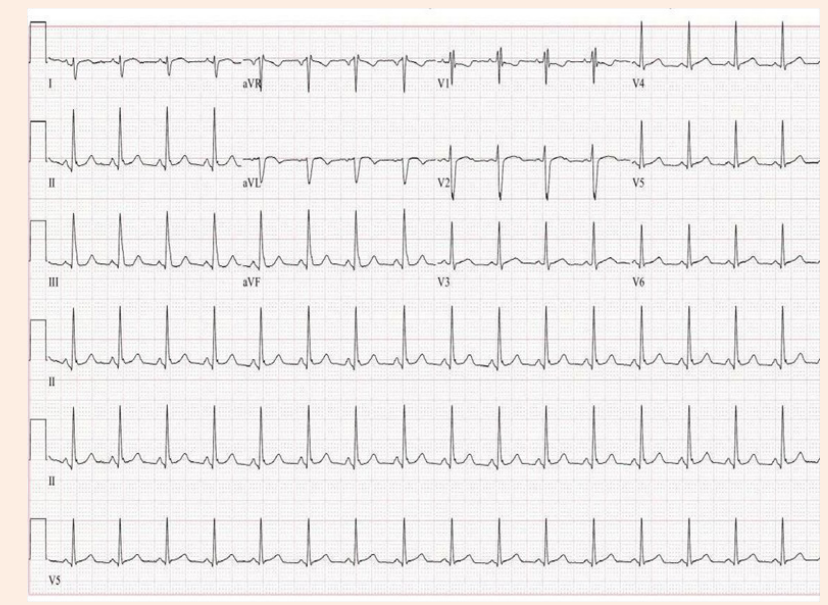

Figure 1: Electrocardiogram (ECG).

Table 1: Laboratory findings.

\begin{tabular}{|c|c|c|}
\hline & Value & Reference Interval \\
\hline WBC & $9 \times 103 / \mu \mathrm{L}$ & $4-10 \times 103 / \mu \mathrm{L}$ \\
\hline RBC & $4.08 \times 106 / \mu \mathrm{L}^{-}$ & $4.1-5.1 \times 106 / \mu \mathrm{L}$ \\
\hline HGB & $11.6 \mathrm{~g} / \mathrm{dl}^{-}$ & $12-16 \mathrm{~g} / \mathrm{dl}$ \\
\hline HCT & $34.8^{-}$ & $36-46 \%$ \\
\hline MCV & $85.3 \mathrm{fL}$ & $79-98 \mathrm{fL}$ \\
\hline Platelet Count & $165 \times 103 / \mu \mathrm{L}$ & $130-400 \times 103 / \mu \mathrm{L}$ \\
\hline D-Dimer & $0.2 \mu \mathrm{m} / \mathrm{ml}$ & $\leq 0.5 \mu \mathrm{g} / \mathrm{ml}$ \\
\hline BUN & $14 \mathrm{mg} / \mathrm{dl}$ & $10-20 \mathrm{mg} / \mathrm{dl}$ \\
\hline Creatinine & $0.71 \mathrm{mg} / \mathrm{dl}$ & $0.57-1.11 \mathrm{mg} / \mathrm{dl}$ \\
\hline Troponin I & $1.125 \mathrm{ng} / \mathrm{ml}$ & $\leq 0.036 \mathrm{ng} / \mathrm{ml}$ \\
\hline TSH & $2.657 \mu \mathrm{IU} / \mathrm{ml}$ & $0.34-4.792 \mu \mathrm{IU} / \mathrm{ml}$ \\
\hline
\end{tabular}

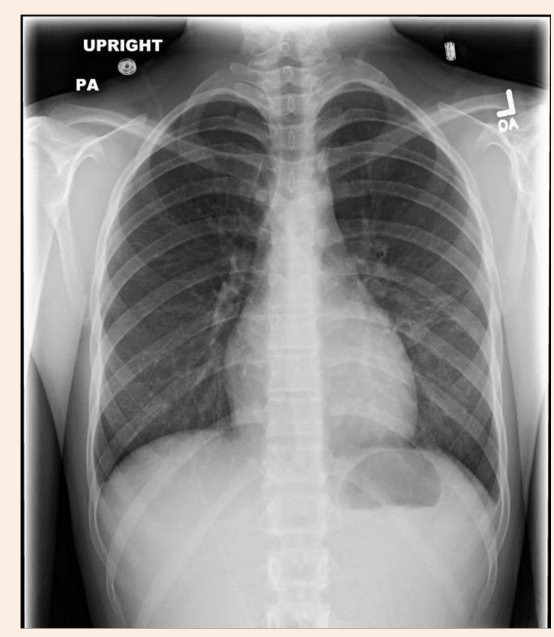

Figure 2: Chest radiography.

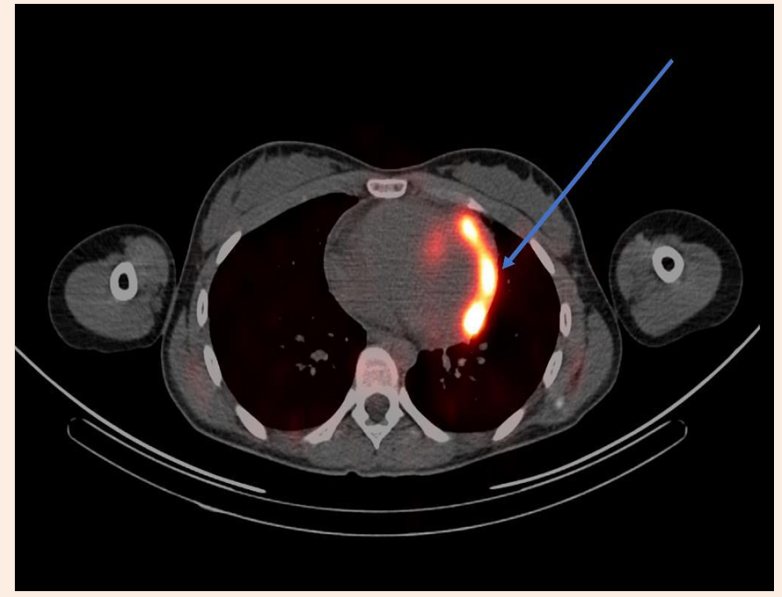

Figure 3: Technetium labeled WBC scan (arrow indicating significant uptake in the left ventricular myocardium).

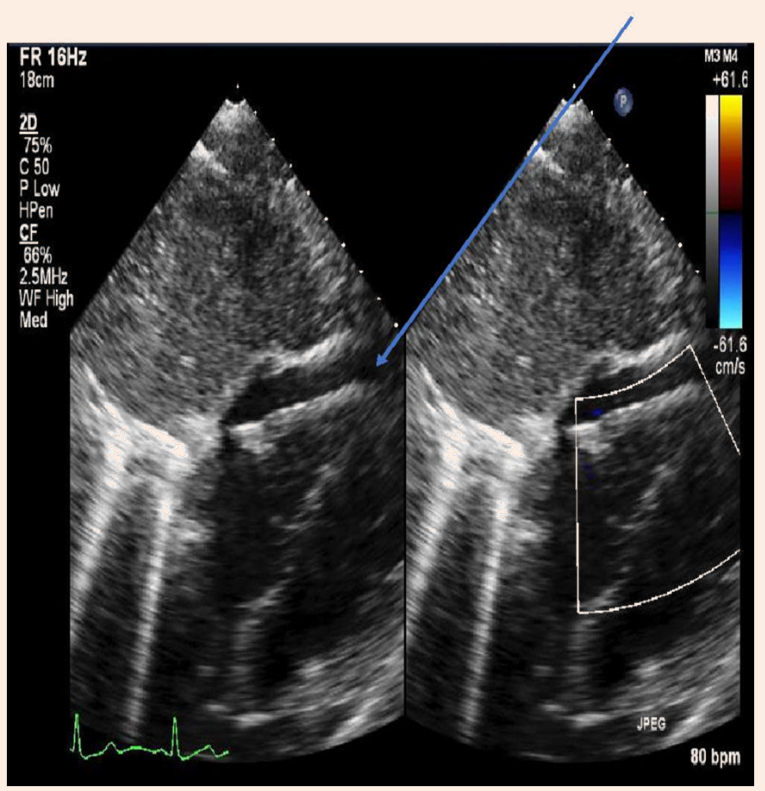

Figure 4: Transthoracic echocardiography (arrow indicating small pericardial effusion).

The patient was treated with ibuprofen $400 \mathrm{mg}$ BID and colchicine $0.6 \mathrm{mg}$ daily and her symptoms improved. However, her fatigue persisted, and she underwent a laparoscopic excisional biopsy of a mesenteric lymph node to exclude lymphoma or another neoplasm. Histopathology of the excised lymph node revealed a preserved lymph node architecture without granulomas or neoplastic cells, but with paracortical hyperplasia. Given the history of myopericarditis, reactive mesenteric lymphadenopathy, fever and chronic fatigue in a young female originally from Turkey, evaluation for FMF was considered. Subsequent genetic testing revealed that the patient was heterozygous for the E148Q mutation in the MEFV gene. Her colchicine dose was increased to $0.6 \mathrm{mg}$ twice daily. She had no further recurrence of myopericarditis and the frequency and severity of abdominal pain 
and high grade fevers were significantly reduced after initiating therapy with colchicine. Serum electrolyte levels, fasting blood glucose level, liver chemistry tests, serum albumin, globulin and total bilirubin levels were all within the respective reference intervals (not shown).

\section{Discussion}

Familial Mediterranean fever (FMF) is an autosomal recessive, hereditary, autoinflammatory disorder caused by mutations in the $M E F V$ gene located on chromosome 16p. The disease is characterized by recurrent episodes of serositis and fever which may be accompanied by an erysipelas-like rash $[3,4]$. FMF is most prevalent in individuals of Ashkenazi Jewish, middle eastern, Turkish and north African descent, with an estimated disease rate in these populations of around one in five hundred and a carrier rate varying between one in eight to one in four [2]. FMF has also been reported in populations, such as Greek and Italian, albeit at a much lower disease prevalence and carrier state [3]. Our patient presented with a six-month history of intermittent right lower quadrant abdominal pain, joint pains and high grade fevers with suspicion for appendicitis requiring an appendectomy. Finally, pleuritic-like chest pain with associated marked troponemia and a technetium labeled WBC scanning revealing neutrophilic inflammation in the left ventricular myocardium and overlying pericardium.

Although myopericarditis is mainly caused by viral infections such as with coxsackie $B$, adenovirus, herpes viruses, hepatitis $C$ virus, parvoviruses and echovirus, among others $[4,5]$, in this case, it was most likely secondary to autoinflammation in the setting of FMF. The clinical presentation of myopericarditis is variable but commonly involves fever, fatigue, weakness, chest pain (usually pleuritic) and possibly a preceding flu-like viral illness. Due to the low yield and lack of targeted therapy, routine serologic testing for viral infections are not routinely performed [6,7]. ECG findings of myopericarditis include transient ST segment and T-wave abnormalities in addition to atrial and ventricular tachyarrythmias [8-12]. Early repolarization, atrioventricular and intraventricular conduction defects may be observed in myopericarditis [8-12] However, the absence of these ECG findings, as in our patient, does not rule out myopericarditis [12]. Laboratory findings that may support the diagnosis of myopericarditis include an elevated white blood cell count, erythrocyte sedimentation rate, C-reactive protein and serum cardiac biomarkers such as troponin I and creatine kinase [8-10]. The diagnosis is usually suggested with a combination of clinical symptoms, elevated inflammatory markers and cardiac biomarkers, typical ECG changes and possible echocardiographic evidence of left ventricular systolic dysfunction. However, other imaging studies (e.g. radionuclide scanning, cardiac MRI) can be used in the diagnosis [4-10,13]

With regard to radionuclide scanning, gallium-67, indium-111, and technetium-99m have been used. Approximately $90 \%$ of gallium-67 is retained in plasma after intravenous injection and almost all of it is bound to transferrin [12]. Gallium-67 also binds to lactoferrin which has high levels in infectious foci $[14,15]$. In myopericarditis, gallium-67 single photon emission commuted tomography (SPECT) scan usually reveals significant uptake in the myocardium and pericardium; however, use of gallium-67 over time has diminished because of its lack of specificity [14]. WBC scanning to diagnose myopericarditis involves using the patient's white blood cells, usually neutrophils, radiolabeled with technetium-99m or indium-111 antimyosin antibody and injected intravenously back into the patient, followed by single photon emission commuted tomography [15]. As with the gallium scan, the WBC scan will usually reveal significant activity in the myocardium and pericardium on SPECT in patients myopericarditis [16]. Both techniques of WBC scanning have been extensively used, specifically the indium-111 antimyosin antibody WBC scan. The sensitivity and negative predictive value of the indium-111 antimyosin antibody in diagnosing myocarditis were 91-100 \% and 93-100 \% in one study [17]. The use of indium-111 antimyosin antibody scan has decreased over time because of radiation exposure and the requisite 48-hour delay in obtaining the imaging results [16]. Our patient underwent a technetium$99 \mathrm{~m}$ labeled WBC scan which revealed intense abnormal uptake in the left ventricular myocardium, with a noted small pericardial effusion.

More recently, cardiac MRI (CMR) has become a key component in the diagnosis of myocarditis [18]. Possible findings in myopericarditis on CMR may include global left ventricular (LV) dysfunction or left ventricular wall motion abnormalities, pericardial effusion, transient increase in myocardial thickness, tissue edema, early gadolinium enhancement indicating hyperemia and capillary leakage, in addition to late gadolinium enhancement indicating necrosis and fibrosis [16]. A definitive diagnosis of myopericarditis, which is unnecessary, would require endomyocardial biopsy (EMB), which is specific but not sensitive given the focal nature of the disease [4-10,13]. We elected to forgo obtaining an endomyocardial biopsy in our patient since the workup that was performed was very suggestive of myopericarditis related to FMF and biopsy would not have altered management.

The mainstay of treatment of myopericarditis in the setting of FMF is to control the patient's underlying autoinflammatory disease with NSAIDs and colchicine [4-10,13]. Colchicine inhibits neutrophil chemotaxis, adhesion and mobilization by causing microtubule depolymerization [19]. Colchicine also inhibits superoxide production in addition to NACHT-LRRPYDcontaining protein 3 (NALP3) inflammasome and interleukin-1 $\beta$ (IL-1 $\beta$ ) [19]. The MEFV gene, which is mutated in FMF, encodes pyrin, a protein involved in the regulation of inflammation [20]. Normally, pyrin indirectly inhibits the formation of NALP3 inflammasome, but an abnormal pyrin protein is unable to carry out this function [21]. Increased levels of IL-1 $\beta$ are also noted in monocytes carrying an $M E F V$ gene mutation [21]. This explains why colchicine, in addition to TNF- $\alpha$ and IL-1 $\beta$ antagonists can suppress inflammation in FMF [21].

Our patient was a heterozygote for the E148Q MEFV mutation. V726A, M694V, M694I, M680I, and E148Q mutations account for the majority of all MEFV gene mutations, with the M694V mutation being the most prevalent mutation in Armenians, Arabs, Jews and Turks [22-24]. Patients who carry one abnormal allele of the MEFV gene are predicted to be asymptomatic, but the FMF phenotype has been reported in heterozygotes [25]. This finding postulates a more complex mode of inheritance [24-26]. 
A definitive diagnosis of FMF based on the Tel-Hashomer clinical criteria requires two or more major criteria or one major plus two minor criteria (Table 2). A probable diagnosis requires one major criterion and one minor criterion [27]. Our patient met two major criteria which included recurrent febrile episodes with serositis in addition to a favorable response to colchicine during attacks (Table 2). She was initially started on ibuprofen $400 \mathrm{mg}$ BID for around 3 weeks after her episode of myopericarditis and was then transitioned to colchicine $0.6 \mathrm{mg}$ daily which was titrated to $0.6 \mathrm{mg}$ BID. In a small subset of patients who do not respond to colchicine, colchicine can be combined with either IL-1 inhibitory agents or TNF- $\alpha$ inhibitory agents in order to try to prevent the most feared complication of FMF, which is secondary amyloidosis [4-10,13]. Disease control involves following the patient for recurrent "FMF attacks", in addition to following inflammatory markers.

Table 2: Tel-Hashomer diagnostic criteria for familial Mediterranean fever (FMF).

\begin{tabular}{|c|}
\hline Major Criteria \\
\hline Recurrent febrile episodes accompanied by serositis \\
\hline AA amyloidosis without predisposing disease \\
\hline Favorable response to colchicine during attacks \\
\hline Minor Criteria \\
\hline Recurrent febrile episodes \\
\hline Erysipelas-like rash \\
\hline FMF in a first degree relative \\
\hline
\end{tabular}

\section{Acknowledgement}

None.

\section{Conflicts of Interest}

None.

\section{References}

1. Ben-Chetrit E, Touitou I (2009) Familial Mediterranean Fever in the world. Arthritis Rheum 61(10): 14471453.

2. Imazio M, Trinchero R (2010) The spectrum of inflammatory myopericardial diseases. Int J Cardiol 144(1): 134.

3. Isabelle Jéru, Véronique Hentgen, Emmanuelle Cochet, Philippe Duquesnoy, Gaëlle Le Borgne, et al. (2013) The Risk of Familia Mediterranean Fever in MEFV Heterozygotes: A Statistical Approach

4. Sarkisian T, Ajrapetian H, Beglarian A, Shahsuvarian G, Egiazarian A (2008) Familial Mediterranean Fever in Armenian population. Georgian Med News 156: 105-111.

5. Spodick DH (2001) Myopericarditis/Perimyocarditis. In: The Pericardium, Marcel Dekker, Inc, New York, USA, pp. 114.

6. Caforio AL, Pankuweit S, Arbustini E, Basso C, Gimeno-Blanes J, et al. (2013) Current state of knowledge on aetiology, diagnosis, management, and therapy of myocarditis: a position statement of the European Society of Cardiology Working Group on Myocardial and Pericardial Diseases. Eur Hear J 34(33): 2636-2648.
7. Kindermann I, Barth C, Mahfoud F, Christian U, Matthias L, et al (2012) Update on myocarditis. J Am Coll Cardiol 59(9): 779-792.

8. Imazio M, Cecchi E, Demichelis B, Chinaglia A, Ierna S, et al. (2008) Myopericarditis versus viral or idiopathic acute pericarditis. Heart 94(4): 498-501.

9. Imazio M, Trinchero $\mathrm{R}$ (2008) Myopericarditis: Etiology, management, and prognosis. Int J Cardiol 127(1): 17-26.

10. Imazio M, Demichelis B, Cecchi E, Belli R, Ghisio A, et al. (2003) Cardiac troponin I in acute pericarditis. J Am Coll Cardiol 42(12): 2144 .

11. Morgera T, Di Lenarda A, Dreas L, Pinamonti B, Humar F, et al. (1992) Electrocardiography of myocarditis revisited: clinical and prognostic significance of electrocardiographic changes. Am Heart J 124(2): 455-467.

12. Eckart RE, Love SS, Atwood JE, Arness MK, Cassimatis DC, et al. (2004) Incidence and follow-up of inflammatory cardiac complications after smallpox vaccination. J Am Coll Cardiol 44(1): 201-205.

13. Bonnefoy E, Godon P, Kirkorian G, Fatemi M, Chevalier P, et al. (2000) Serum cardiac troponin I and ST-segment elevation in patients with acute pericarditis. Eur Heart J 21(10): 832.

14. Love C, Palestro CJ (2004) “Radionuclide Imaging of Infection.” J Nucl Med Technol 32(2): 47-57.

15. Hung MY, Hung MJ, Cheng CW (2007) Use of Gallium 67 Scintigraphy to Differentiate Acute Myocarditis from Acute Myocardial Infarction. Tex Heart Inst J 34(3): 305-309.

16. Narula J, Khaw BA, Dec GW, Palacios IF, Newell JB, et al. (1966) Diagnostic accuracy of antimyosin scintigraphy in suspected myocarditis. J Nucl Cardiol 3(5): 371-381.

17. Jeserich M, Konstantinides S, Pavlik G, Bode C, Geibel A (2009) Noninvasive imaging in the diagnosis of acute viral myocarditis. Clin Res Cardiol 98(12): 753-763.

18. Friedrich MG, Sechtem U, Schulz-Menger J, Holmvang G, Alakija $P$, et al. (2017) Cardiovascular Magnetic Resonance in Myocarditis: A JACC White Paper. J Am Coll Cardiol 53(17): 1475-1487.

19. Leung YY, Yao Hui LL, Kraus VB (2017) Colchicine --- update on mechanisms of action and therapeutic uses. Semin Arthritis Rheum 45(3): 341-350.

20. Özen S, Batu ED, Demir S (2017) Familial Mediterranean Fever: Recent Developments in Pathogenesis and New Recommendations for Management. Front Immunol 8: 253.

21. Campbell L, Raheem I, Malemud CJ, Askari AD (2006) The Relationship between NALP3 and Autoinflammatory Syndromes. Int J Mol Sci 17(5).

22. (1997) Ancient missense mutations in a new member of the RoRet gene family are likely to cause familial Mediterranean fever. The International FMF Consortium. Cell 90(4): 797-807.

23. Touitou I (2001) The spectrum of Familial Mediterranean Fever (FMF) mutations. Eur J Hum Genet 9(7): 473-483.

24. Booty MG, Chae JJ, Masters SL, Remmers EF, Barham B, et al. (2009) Familial Mediterranean fever with a single MEFV mutation: where is the second hit? Arthritis Rheum 60(6): 1851-1861.

25. French FMF Consortium (1997) A candidate gene for familial Mediterranean fever. Nat Genet 17(1): 25-31. 
26. Padeh S, Shinar Y, Pras E, Zemer D, Langevitz P, et al. (2003) Clinica and diagnostic value of genetic testing in 216 Israeli children with Familial Mediterranean fever. J Rheumatol 30(1): 185-190.
27. Bashardoust B (2015) Familial Mediterranean fever; diagnosis, treatment, and complications. J Nephropharmacol 4(1): 5-8. 